\title{
Modulated photothermal measurements applied to multi-layer superinsulation foils
}

\author{
by Haj-Daoud ${ }^{1,2,3}$, I. Delgadillo-Holtfort ${ }^{1,4}$, V. John ${ }^{5}$, B.K. Bein ${ }^{1}$, \\ N. Marquardt ${ }^{5}$ and J. Pelzl ${ }^{1}$
}

\author{
${ }^{1}$ Exp.Phys. III, Solid State Spectroscopy, Ruhr-University, D-44780 Bochum, Germany \\ E-mail: delgadillo@ep3.ruhr-uni-bochum.de \\ ${ }^{2}$ supported by DAAD, \\ ${ }^{3}$ Palestine Academy for Science and Technology, P.O. Box 3637, Al-Bireh, Palestine \\ ${ }^{4}$ on leave from CINVESTAV-IPN, Depto. Física, 07000 MEXICO D.F \\ ${ }^{5}$ Accelerator Physics and Synchrotron Radiation (IBS), University of Dortmund, D-44221 Dortmund, \\ Germany
}

\begin{abstract}
Photothermal measurements based on modulated heating in the visible spectral range and IR detection of the thermal response are used to determine the effective thermal transport properties and the shielding properties of multi-layer superinsulation foils consisting of different numbers of aluminized mylar layers and spacer layers. The measurements have been run at ambient temperature, both at ambient pressure and at reduced pressures between $1 \mathrm{mbar}$ and $300 \mathrm{mbar}$.
\end{abstract}

\section{Introduction}

Owing to voids, an effective low mass density and a relatively low thermal conductivity, the internal radiative heat transport can play an important role in heat insulation materials. Apart from foam materials and ceramics such effects are also relevant in multi-layer superinsulation foils consisting of highly reflective aluminized mylar foils, used for thermal insulation of space shuttles and satellites in space-flight or of superconducting magnetic coils.

In large scale applications of superconductivity in particle accelerators, e.g. in the Large Hadron Collider at CERN, the energy costs for cooling are relatively high and require optimization of the insulation system. This may be achieved by an appropriate number of insulation and spacer layers, by optimizing the thickness and homogeneity of the individual mylar layer, by optimizing the thickness and the reflection properties of the metal films, and by searching for a suitable material for metallization.

Photothermal measurements based on modulated heating in the visible spectrum and IR detection of the thermal wave response are used here to determine the effective thermal transport properties, respectively the shielding properties of multi-layer superinsulation foils consisting of different numbers of aluminized mylar layers and spacer layers. The measurements have been run at ambient temperature, both at ambient and at reduced pressures.

In chapter 2, the measurement method is briefly described. Subsequently the results obtained at ambient pressure for different numbers of aluminized mylar foils and spacer layers of the superinsulation system Lydall Dam are presented (chapt. 3). The results obtained at reduced pressures (1 mbar - 300 mbar) for a constant number of aluminized mylar layers are presented in chapter 4. The measured data are interpreted in the frame of a photothermal theory, considering both conductive and radiative heat transport.

\section{Infrared radiometry of thermal waves}

The photothermal measurements and their interpretation are based on the concept of transmitted thermal waves [1], where the sample's front surface is heated and where the thermal response is detected at the rear surface. This method is especially suited to measure the insulation properties of samples of finite thickness and of layer systems with different numbers of layers.

The measuring system developed for the IR detection of thermal waves consists of four 
main components (Figure 1): an Argon ion laser (Spectra Physics, Series 2000) to excite thermal waves of small temperature amplitude, a vacuum cell to keep the samples at a desired constant sample temperature and constant pressure, a MCT detector to measure the emitted radiation, and a Lock-in amplifier to filter the small periodical variations of IR emission, corresponding to the thermal wave $\Delta T(f)$ from the comparatively large radiation background related to the stationary sample temperature $T_{\mathrm{s}}$.

For the present measurements, the samples have been kept at $20^{\circ} \mathrm{C}$ and the pressure has been varied between $1 \mathrm{mbar}$ and ambient pressure. The low pressure limit of the cell has been found at about $10^{-4} \mathrm{mbar}$. The laser beam of a nominal power of $1000 \mathrm{~mW}$ has been modulated with the help of an acousto-optic modulator (Isle Optic, LM 080) in the frequency interval from $0.03 \mathrm{~Hz}$ to $20 \mathrm{~Hz}$. A large heating spot of about $10 \mathrm{~mm}$ has been used, to reduce the lateral heat transport parallel to the metallized foils.

For the detection of the IR emission a photoconductive HgCdTe detector (Judson IR) and IR optics consisting of two $\mathrm{BaF}_{2}$ lenses and a $\mathrm{Ge}$ cut-on filter are used which allow a detection interval of $1 \mu \mathrm{m}<\lambda<12 \mu \mathrm{m}$ in the near and mid infrared and a maximum focussed solid angle of $\Omega_{\mathrm{D}}=0.196$ steradians. Based on this detection system thermal wave amplitudes of only $\Delta T=44 \mu \mathrm{K}, 15 \mu \mathrm{K}$ and $9 \mu \mathrm{K}$ have been detected at stationary sample temperatures of $T=300 \mathrm{~K}, 400 \mathrm{~K}$ and $500 \mathrm{~K}[2,3]$.

For the quantitative interpretation, the present measurements, which are based on the transmission of thermal waves, have been calibrated with the help of reference data, measured for the heated sample support or samples of a different number of insulating layers. Assuming IR opaque samples, the normalized amplitudes $S_{n}$ are described by [1]

$$
S_{n}=\left(\frac{S_{s}}{S_{r}}\right)=\left(\frac{\eta_{s} e_{r}}{\eta_{r} e_{s}}\right)\left[\frac{\exp \left(2 \sqrt{\pi f \tau_{r}}\right)-2 \cos \left(2 \sqrt{\pi f \tau_{r}}\right)+\exp \left(-2 \sqrt{\pi f \tau_{r}}\right)}{\exp \left(2 \sqrt{\pi f \tau_{s}}\right)-2 \cos \left(2 \sqrt{\pi f \tau_{s}}\right)+\exp \left(-2 \sqrt{\pi f \tau_{s}}\right)}\right]^{1 / 2}
$$

where $S_{s}$ is the signal measured for the foils of unknown properties and $S_{\mathrm{r}}$ the signal measured for the reference. The quantities $\eta, e$, and $\tau$ in equ. (1) represent the photothermal efficiency, thermal effusivity, and the thermal diffusion time, respectively, and the indexes $s$ and $r$ refer to the sample of unknown properties and to the reference, respectively. The photothermal efficiency $\eta$ is defined as the fraction of the incident radiative flux which is not reflected, and the thermal effusivity $e=\sqrt{k \rho c}$ is a combined thermophysical parameter, which is a measure for transient surface heating processes and for the heat transition between layers of different thermal properties. The thermal diffusion time of reference and sample, respectively, is given by

$$
\tau_{r, s}=d_{r, s}^{2} / \alpha_{r, s}
$$

where $d$ is the sample thickness and $\alpha$ the effective thermal diffusivity, which is defined by $\alpha=k /(\rho c)$. The quantities $k, \rho$, and $c$ are the thermal conductivity, mass density, and specific heat capacity, respectively. In the low frequency limit, equ. (1) gives information on the damping of the steady state heat transport based on radiation and conduction by the foil system. The slope of the normalized amplitudes at the higher frequencies, which can be approximated by

$$
\ln \left(S_{n}\right)=\ln \left(S_{s} / S_{r}\right) \approx \ln \left[\left(\eta_{s} e_{r}\right) /\left(\eta_{r} e_{s}\right)\right]+\pi^{1 / 2}\left(\sqrt{\tau}_{r}-\sqrt{\tau}_{s}\right) f^{1 / 2}
$$

gives the possibility to derive the effective thermal diffusivity from the measured amplitude signals. For the relative changes of the thermal diffusivity, a more reliable interpretation can be obtained when the normalized phase signals are considered,

$$
\tan \left(\Phi_{s}-\Phi_{r}\right)=\frac{\tan \left(\Phi_{s}\right)-\tan \left(\Phi_{r}\right)}{1+\tan \left(\Phi_{s}\right) \tan \left(\Phi_{r}\right)}=\frac{\tan \left(\sqrt{\pi f \tau_{s}}\right) \cot \left(\sqrt{\pi f \tau_{s}}\right)-\tanh \left(\sqrt{\pi f \tau_{r}}\right) \cot \left(\sqrt{\pi f \tau_{r}}\right)}{1+\tan \left(\sqrt{\pi f \tau_{s}}\right) \cot \left(\sqrt{\pi f \tau_{s}}\right) \tanh \left(\sqrt{\pi f \tau_{r}}\right) \cot \left(\sqrt{\pi f \tau_{r}}\right)}
$$

the interpretation of which depends on a smaller number of parameters, namely only on the thermal diffusion times of the reference and sample. Additionally, some measurements have been interpreted using a more general thermal wave theory where both conductive and radiative heat transport are considered explicitly $[4,5]$. 


\section{Measurements at ambient pressure}

Subsequently we present the results of thermal wave measurements of the effective thermal transport properties of multi-layer superinsulation foils for the example of the multilayer system Lydall DAM. The multi-layer system Lydall DAM consists of externally metallized mylar foils of $25 \mu \mathrm{m}$ thickness both at the front and rear of the multi-layer system. In the interior of the multi-layer system, spacer layers and metallized mylar foils of $6 \mu \mathrm{m}$ thickness follow each other alternately. Our measurements have been run on an external foil separately and on different multi-layer systems consisting of the external foil at the front surface and up to seven sequences of spacer and internal layers. First, the results measured at ambient pressure are discussed.

As Figure 2 shows, the IR transmission signals, which have been measured at the rear surface of the samples, decrease with the increasing number of insulating layers. The signals ( $\square$ ) separately measured for the external foil $(25 \mu \mathrm{m}$ thick) are above the noise limit (*) over the whole measured frequency range $[0.03 \mathrm{~Hz}, 20 \mathrm{~Hz}]$. The signals, measured for the samples containing an increasing number of spacer and internal layers $(\mathbf{x}, \mathbf{+}, \Delta) \square$, respectively an additional external foil $(25 \mu \mathrm{m})$ at the rear surface $(\diamond)$ reach the noise limit (*) already at about $10 \mathrm{~Hz}, 4 \mathrm{~Hz}, 2 \mathrm{~Hz}$, and $1 \mathrm{~Hz}$.

In part the observed frequency dependence of the measured raw signals is due to the frequency characteristics of the used electronic equipment, which can be eliminated by calibration of the signals with the help of reference signals, e.g. by normalizing the signals measured for the various insulation foils by the signal $(\mathbf{0})$ measured for the heated sample support (Figure 2).

In the low frequency limit, the normalized amplitudes (Figure 3) give information on the attenuation of the incident steady state heat flux by the various foils. Thus, the heat flux is reduced by the external $25 \mu \mathrm{m}$ thick metallized foil to a value of only $5 \%$ ( $\square$ ) $\square$, whereas for the composite insulations with the increasing number of spacer and internal layers $(\mathbf{x},+, \Delta)$ the steady state heat transport is reduced to values of about $4 \%, 3.5 \%$, and $3 \%$. For the sample with the additional external foil at the rear surface $(\diamond)$, it is reduced to about $2.5 \%$.

In Figure 4 the normalized phases are shown in comparison with theoretical approximations according to equ. (4), giving information on the effective thermal diffusivity, decreasing with the number of insulation layers according to the ratio: $0.49: 0.41: 0.19: 0.13: 0.08$. From the general agreement between measured phases and theoretical solutions, we can conclude, that the main contributions to the heat transport are diffusive and that only the slight deviations at higher frequencies can be identified as radiative contributions.

This in shown in Figure 5, where the normalized phases measured for the external metallized mylar foil and a sandwich system consisting of an external foil, a spacer layer and an internal layer are compared with theoretical solutions of the thermal wave theory for IR translucent materials $[4,5]$. The continous curves, in perfect agreement with the measured data at low frequencies, are calculated for an IR opaque sample. The broken curves and the broken-pointed curves are calculated for decreasing IR absorption coefficients and approach the measured data at the higher frequencies.

\section{Measurements at reduced pressures}

The first measurements at reduced air pressures have been applied at a sandwich system consisting of the external foil, one spacer and one internal layer. Thus the amplitude data obtained at reduced pressure (Figure 6) can be compared with the data represented by the symbol $\mathbf{x}$ in Figure 3 . In order to calibrate the measured data, the signals measured for the heated sample support at reduced pressures have been used. As can be seen from the low frequency limit in Figure 6, the attenuation of the incident steady state heat flux has changed from about 0.037 at ambient pressure to about 0.020 at a pressure of 1 mbar.

In Figure 7 and 8, the normalized phases for different pressures are shown. In Figure 7, the higher pressures, 750 mbar $(\boldsymbol{\nabla}), 50 \mathrm{mbar}(\Delta)$ and $20 \mathrm{mbar}(\square)$, are compared, whereas 
in Figure 8 the lower pressures are compared: 20 mbar ( $\square)$, 3 mbar (+) and 1.5 mbar (x). In general, the normalized phases decrease with decreasing pressure, which means that the effective thermal diffusivity also decreases with further decreasing pressure. This is in agreement with the stronger attenuation of the transmitted heat flux observed in Figure 6 . Between ambient pressure and about 20 mbar, the slope of the normalized phases and thus the thermal diffusivity decreases only slowly, whereas below 20 mbar the decrease of the thermal diffusivity is stronger. Additionally, we observe, that the phases start first to decay at the higher frequencies (Figure 7), corresponding to lower thermal diffusion length.

At the lower pressures, the detection limits of the transmission signals shift to lower frequencies. According to Figure 6 , at a pressure of 1 mbar the detection limit is already at 4 $\mathrm{Hz}$ for the system consisting of external foil, spacer layer and internal layer.

\section{Conclusions and outlook}

The measurements, both at ambient and reduced pressures, show that the steady-state heat flux attenuation factor of superinsulation foils can be determined by extrapolation from the low frequency limit of the thermal wave amplitudes. The relative decrease of the thermal diffusivity can be determined from the intermediate frequency range. These two quantities, the steady-state attenuation factor and the effective thermal diffusivity, are the relevant parameters to characterize the efficiency of thermal insulation foils. Difficulties due to background radiation, always present in steady-state measurements, are here overcome by the modulation technique.

In order to determine the absolute values of the thermal diffusivity, reference measurements of samples of well defined IR optical and thermal properties have still to be done. As shown in this paper, the measurements can be interpreted based on the theory of thermal waves transmitted through samples of limited thickness. The radiative contributions to the heat transport have been identified only at higher modulation frequencies. This is due to the fact that the thermal contact between the various mylar foils is relatively narrow.

With decreasing air pressure, below about 20 mbar the increase of the steady-state attenuation factor and the decrease of the thermal diffusivity are more pronounced.

Further measurements to compare the efficiency of different superinsulation foils are in progress. Future measurements will have to be done both at further reduced pressures and reduced temperatures. In order to cope with cryo-applications, larger IR wavelengths available as edge radiation in Synchrotrons will have to be used for the excitation of thermal waves. As the used measuring technique is absolutely non-contact and allows remote detection, this can easily be achieved.

The technique of the present work, applied here in the transmission configuration of thermal waves to compare different superinsulation foil systems, can also be used for the efficiency control of multi-layer superinsulation systems under working conditions. In this case, the thermal wave technique has to be applied in the reflection configuration, where the thermal waves are excited and detected at the same surface. For such measurements, the surface has to be accessible to the modulated heating source and to a sufficiently large detecion angle, e.g. using two lines of sight, one for laser heating and one for IR detection.

\section{References}

[1] B.K. Bein, J. Gibkes, A. Mensing, J. Pelzl, High Temp.-High Press. 26 (1994), 299-307.

[2] J. Bolte, B.K.Bein, J.Pelzl, Quantitative InfraRed Thermography QIRT'96, (eds. D. Balageas, G.Busse, C.M.Carlomagno), Eurotherm Series 50, Edizioni ETS Pisa (1997), 9-14.

[3] J. Bolte, J.H. Gu, B.K. Bein, High Temp.- High Pressures 29 (1997), 567-580.

[4] A.Haj Daoud, "Photothermal materials characterization at higher temperatures by means of IR radiometry", PhD thesis, Ruhr-Universität Bochum (1999).

[5] A. Haj-Daoud, D. Dietzel, F. Macedo, I. Delgadillo-Holtfort, B.K. Bein, "Theory of thermal waves in IR transparent bodies", to be published 2000. 


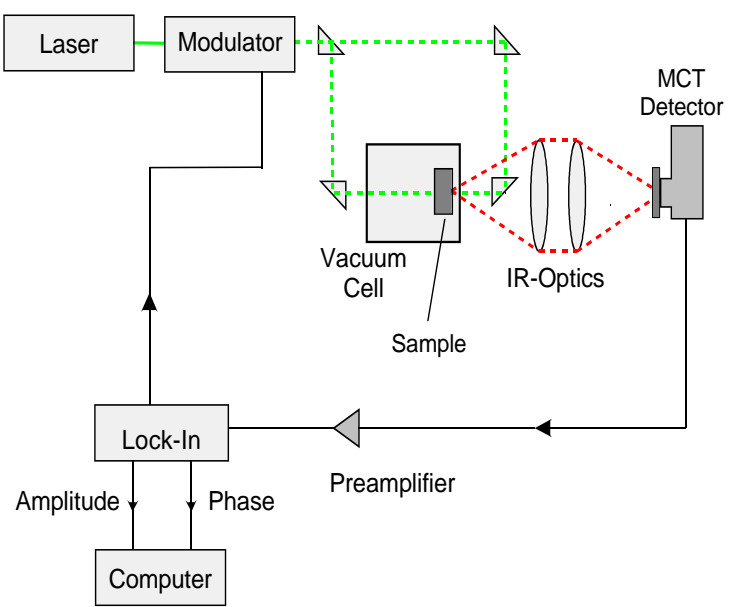

Figure 1: Schematic of thermal wave detec-tion in a vacuum cell, both in the transmission configuration and the reflection configuration of thermal waves.

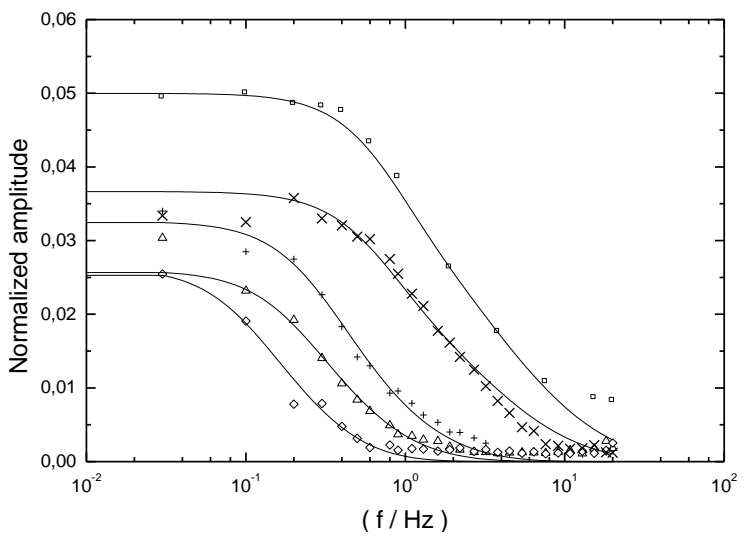

Figure 3: Normalized amplitudes of the samples with increasing number of insulation layers, in comparison with theoretical solutions according to equ. (1):

$\square$ external foil $(25 \mu \mathrm{m})$

$\mathbf{x}$ ext. foil + spacer + internal foil $(6 \mu \mathrm{m})$

+ ext. foil $+3 \times($ spacer + internal foil $)$

$\Delta$ ext. foil $+5 \times($ spacer + internal foil $)$

$\diamond$ ext. foil $+7 \times$ (spacer + int. foil $)+$ ext. fl.

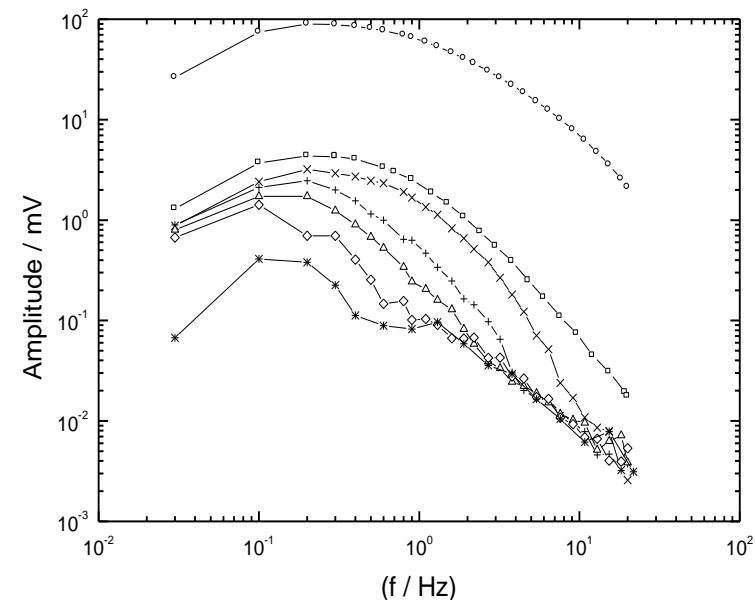

Figure 2: Transmission signals measured for the heated sample support (0) and signal attenuation measured for multilayer systems consisting of Lydall DAM sublayers:

$\square$ external foil $(25 \mu \mathrm{m})$

$\mathbf{x}$ ext. foil + spacer + internal foil $(6 \mu \mathrm{m})$

+ ext. foil $+3 \times$ (spacer + internal foil)

$\Delta$ ext. foil $+5 \times($ spacer + internal foil $)$

$\diamond$ ext. foil $+7 x$ (spacer + int. foil $)+$ ext. fl.

$*$ noise signal

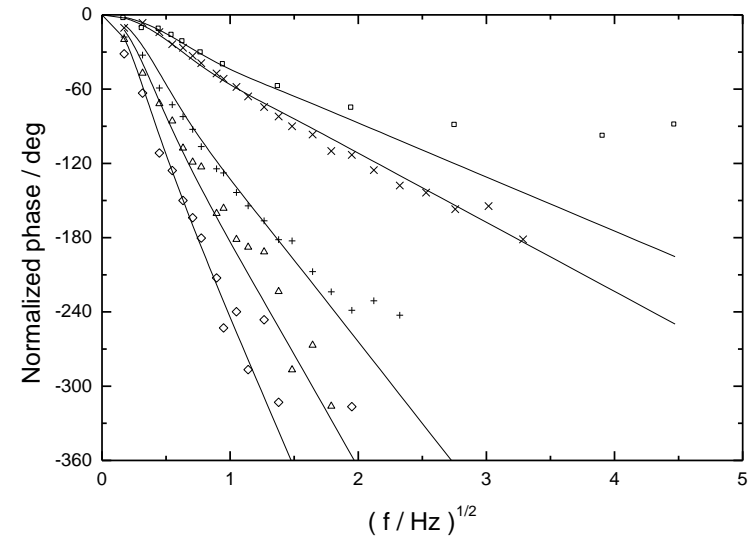

Figure 4: Normalized phases in comparison with theoretical approximations according to equ. (4):

$\square$ external foil

x external foil + (spacer + internal foil)

+ ext. foil $+3 \times$ (spacer + internal foil $)$

$\Delta$ ext. foil $+5 \times($ spacer + internal foil $)$

$\diamond$ ext. foil $+7 \times$ (spacer + int. foil $)+$ ext. fl. 


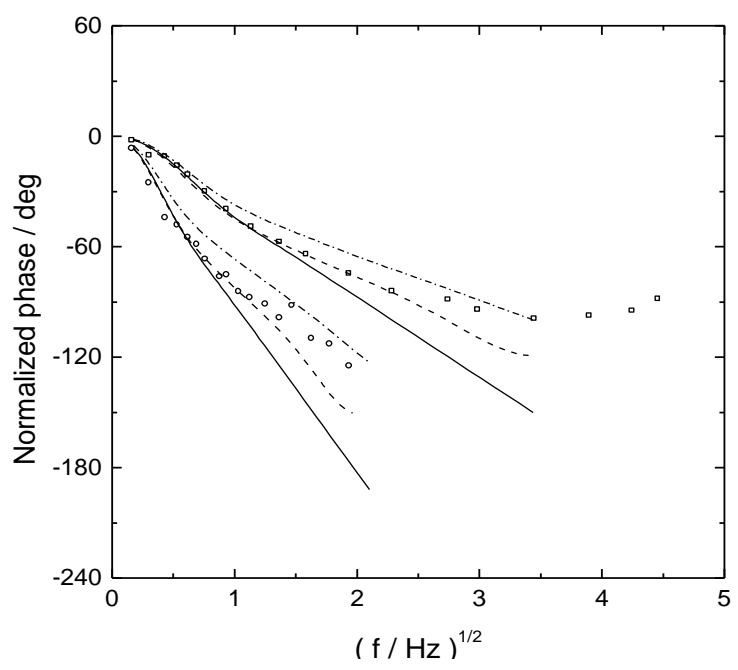

Figure 5: Normalized phases in comparison with approximations based on the thermal wave theory for a slightly IR translucent body.

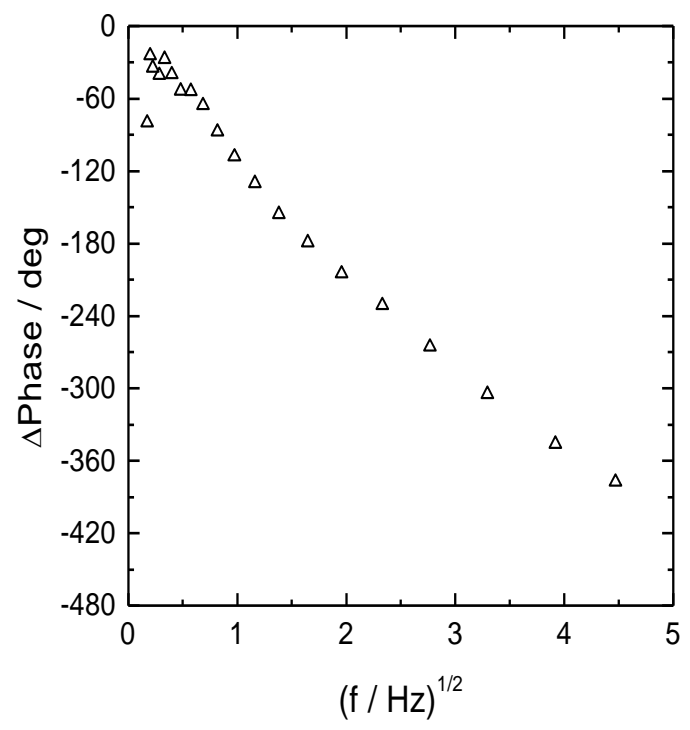

Figure 7: Normalized phases of the sandwich system consisting of external foil, spacer layer and internal foil at reduced pressures:

750 mbar ( $\square), 50$ mbar $(\Delta), 20$ mbar ( $\square)$.

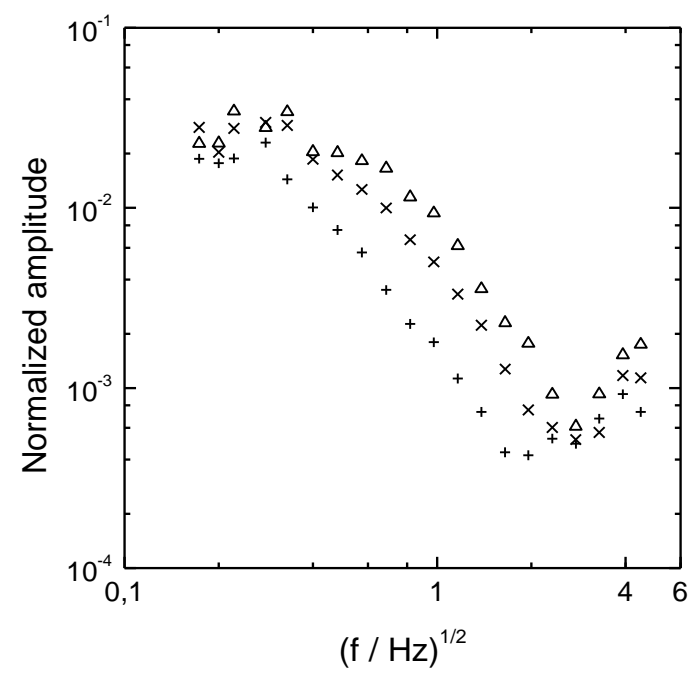

Figure 6: Normalized amplitudes of a sandwich system consisting of external foil, spacer layer and internal foil, measured at reduced pressures: $20 \mathrm{mbar}(\Delta)$, 6 mbar (x), and 1 mbar (+).

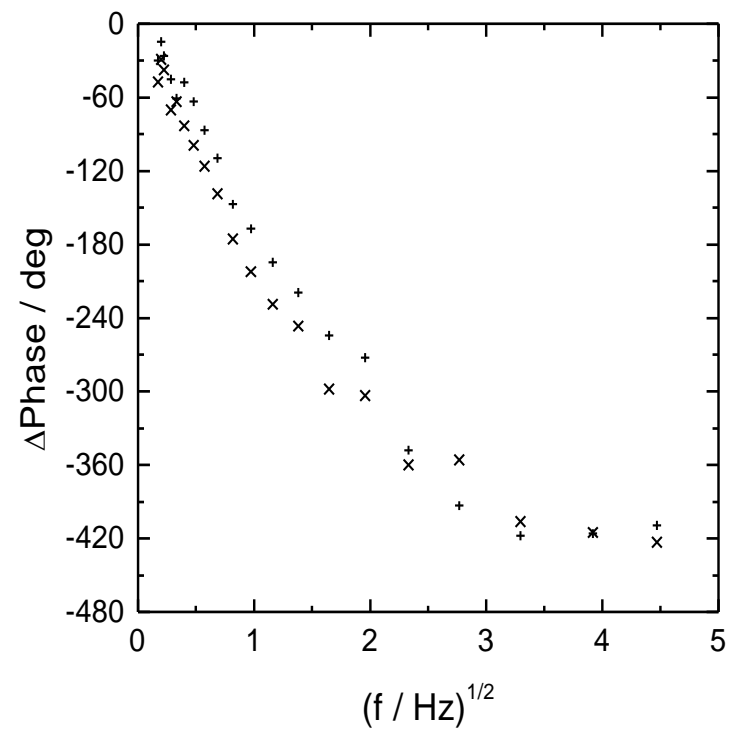

Figure 8: Normalized phases of the sandwich system consisting of external foil, spacer layer and internal foil at reduced pressures:

20 mbar ( $\square$ ), 3 mbar (+), 1.5 mbar (x). 